\title{
Is computed tomographic colonography effective for colorectal cancer screening?
}

Pickhardt PJ, Choi JR, Hwang I, Butler JA, Puckett ML, Hildebrandt $\mathrm{HA}$, et al. Computed tomographic virtual colonoscopy to screen for colorectal neoplasia in asymptomatic adults. N Engl J Med 2003; 349(23):2191-200.

Background: The majority of colorectal cancers arise from benign neoplasms (adenomatous polyps). Adenomas of $1 \mathrm{~cm}$ or more in diameter confer a higher risk of colorectal cancer than smaller ones. The goals of colorectal cancer screening in asymptomatic individuals are to detect cancers and to identify and remove adenomatous polyps. In 2001 the Canadian Task Force on Preventive Health Care endorsed fecal occult blood testing or flexible sigmoidoscopy, or both, as initial screening tests in people 50 years of age and older with average risk of disease. ${ }^{1}$ The evidence was judged insufficient to recommend or oppose colonoscopy for use as the initial screening test. Virtual colonoscopy, or CT colonography, is a new method for colorectal cancer screening that involves a colonic preparation but not conscious sedation or recovery time for the patient.

Design: A total of 1233 asymptomatic adults (mean age 57.8 years) $\&$ underwent same-day virtual and optical colonoscopy at 3 US medical centres. The patients underwent standard colonic preparation and consumed $500 \mathrm{~mL}$ of barium to tag residual stool and $120 \mathrm{~mL}$ of gastrografin to opacify residual fluid in the lumen. CT scanning was performed with state-of-theart multidetectors using a 3dimensional protocol. The scans were interpreted by experienced radiologists. Optical colonoscopies were performed by experienced endoscopists who were initially unaware of the results of the virtual colonoscopy. All polyps were removed and submitted for pathological examination. After each segment of the colon was visualized, a study coordinator revealed the results of the virtual colonoscopy for that segment. If a polyp measuring $5 \mathrm{~mm}$ or more was seen during the virtual colonoscopy but not during the optical colonoscopy, the endoscopist re-examined the segment. This "segmental unblinding" created an enhanced reference standard against which the results of the 2 types of colonoscopy were compared.

Results: The performance characteristics of the virtual and optical colonoscopy used in this study are summarized in Table 1.

Commentary: The results of this study indicate that the likelihood of missing a clinically significant adenoma using virtual colonoscopy is very low. This means that, in many asymptomatic patients undergoing colorectal cancer
Table 1: Ability of CT virtual colonoscopy and optical (traditional) colonoscopy to detect adenomas

\begin{tabular}{lccc}
\hline & \multicolumn{3}{c}{ Size of lesion } \\
\cline { 2 - 4 } Variable & $\geq 6 \mathrm{~mm}$ & $\geq 8 \mathrm{~mm}$ & $\geq 1 \mathrm{~cm}$ \\
\hline Virtual colonoscopy & & & \\
Sensitivity, \% (and 95\% Cl) & $88.7(82.9-93.1)$ & $93.9(86.3-98.0)$ & $93.8(82.8-98.7)$ \\
Specificity, \% (and 95\% Cl) & $79.6(77.0-82.0)$ & $92.2(90.5-93.7)$ & $96.0(94.8-97.1)$ \\
Positive LR & 4.35 & 11.32 & 23.64 \\
Negative LR & 0.14 & 0.12 & 0.07 \\
Optical colonoscopy & & & \\
Sensitivity, \% (and 95\% Cl) & $92.3(87.1-95.8)$ & $91.5(83.2-96.5)$ & $87.5(74.8-95.3)$ \\
\hline
\end{tabular}

Note: $\mathrm{Cl}=$ confidence interval, $\mathrm{LR}=$ likelihood ratio. screening, optical colonoscopy could be avoided. The number of colonoscopies that would not be needed for polypectomy following virtual colonoscopy would depend on the size threshold chosen for the lesion found. For example, with a threshold of $1 \mathrm{~cm}$ or more, only 1 out of every 13 patients would be advised to undergo optical colonoscopy following virtual colonoscopy. However, research is needed to evaluate the generalizability of these results and to evaluate the clinical outcomes associated with different screening strategies so that evidence-based guidelines can be developed. It is important to recognize that polyps cannot be removed during virtual colonoscopy. Therefore, repeated virtual colonoscopies would be advised for patients who do not proceed to optical colonoscopy. Careful consideration of radiation exposure would be required. Finally, costs need to be evaluated, including those incurred by diagnostic work-up and treatment of (incidental) extracolonic findings, such as renal lesions and abdominal aortic aneurysms. ${ }^{2}$

Practice implications: Despite these impressive results, CT virtual colonoscopy is an evolving technology. It is premature to recommend it for colorectal cancer screening in clinical practice. Its potential role in an evidencebased practice guideline requires further investigation.

Linda Rabeneck

Director, Division of

Gastroenterology

University of Toronto

Toronto, Ont.

\section{References}

1. Colorectal cancer screening: recommendation statement from the Canadian Task Force on Preventive Health Care. CMA7 2001;165(2):206-8.

2. Edwards JT, Wood CJ, Mendelson RM, Forbes GM. Extracolonic findings at virtual colonoscopy: implications for screening programs. Am $\mathcal{F}$ Gastroenterol 2001;96:3009-12. 